Canadian

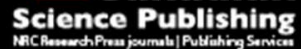

Canadian Journal of Physiology and Pharmacology Revue canadienne de physiologie et pharmacologie

\title{
Effects of lactulose and silymarin on liver enzymes in cirrhotic rats.
}

\begin{tabular}{|r|l|}
\hline Journal: & Canadian Journal of Physiology and Pharmacology \\
\hline Manuscript ID & cjpp-2016-0454.R1 \\
\hline Manuscript Type: & Article \\
\hline Date Submitted by the Author: & 06-Sep-2016 \\
\hline Complete List of Authors: & $\begin{array}{l}\text { Ghobadi Pour, Mozhgan; Bu Ali Sina University } \\
\text { Mirazi, Naser; Bu Ali Sina University Faculty of Science } \\
\text { Alaei, hojjatallah; Isfahan University of Medical Sciences, physiology } \\
\text { Moradkhani, Shirin; Hamadan University of Medical Sciences Medical } \\
\text { School } \\
\text { Rajaei, Ziba; Isfahan University of Medical Sciences } \\
\text { Monsef Esfahani, Alireza; Hamadan University of Medical Sciences Medical } \\
\text { School }\end{array}$ \\
\hline Keyword: & \begin{tabular}{l} 
Liver Cirrhosis; Rat; Silymarin; Thioacetamide. \\
\hline
\end{tabular} \\
\hline
\end{tabular}




\section{Effects of lactulose and silymarin on liver enzymes in cirrhotic 1}

rats.

Mozhgan Ghobadi Pour ${ }^{\mathrm{a}}$, Naser Mirazi*a ${ }^{\mathrm{a}}$, Hojjatollah Alaei ${ }^{\mathrm{b}}$, Shirin 3

Moradkhani $^{\mathrm{c}}$, Ziba Rajaei ${ }^{\mathrm{b}}$, Alireza Monsef Esfahani ${ }^{\mathrm{d}}$. 4

5

${ }^{\mathrm{a}-D e p a r t m e n t}$ of Biology, Faculty of Basic Sciences, Bu-Ali Sina University, Shahid Mostafa 6

Ahmadi Roshan Boulevard, Hamedan, Iran. M ghobadipour90@basu.ac.ir, 7

Mirazi@basu.ac.ir. Phone number: +989188125741 Fax number: +988138381058 8

${ }^{b-D e p a r t m e n t ~ o f ~ P h y s i o l o g y, ~ F a c u l t y ~ o f ~ M e d i c i n e, ~ I s f a h a n ~ U n i v e r s i t y ~ o f ~ M e d i c a l ~ S c i e n c e s, ~} 9$

Hezar-Jerib Ave, Isfahan, Iran.alaei@med.mui.ac.ir, rajaeiz@med.mui.ac.ir $\quad 10$

${ }^{c-}$ Department of Pharmacognosy, Faculty of Pharmacy, Hamadan University of Medical 11

Sciences, Shahid Fahmideh Boulevard, in front of Mardom park, Hamadan, Iran. 12

sh.moradkhani@umsha.ac.ir 13

${ }^{d-}$ Department of Pathology, Hamadan University of Medical Sciences, Shahid Fahmideh 14

Boulevard, in front of Mardom park, Hamadan, Iran. Monsef1340@gmail.com 15 
Silymarin (SM), a mixture of anti-hepatotoxic flavonolignans is being used for liver diseases and lactulose (LAC), a non-absorbable synthetic disaccharide were used in this study to analyze their probable synergic effects on hepatic cirrhotic rat model to see whether they

Keywords: Liver Cirrhosis; Rat; Silymarin; Thioacetamide. 
Cirrhosis is a result of chronic liver injury defined by development of regenerative nodules

Thioacetamide induces hepatotoxicity, originally used as fungicide. Earlier literature suggests acetylimiodolysine derivatives, is responsible for TAA-induced hepatotoxic effects (Kumar

At present, one of the therapeutic strategies for liver fibrosis includes complementary and

We shouldn't consider cirrhosis as the irreversible end stage of chronic liver disease, but rather as a stage in an active process whose advance course(progression or reversion of

Milk thistle (Silybum marianum) has been the dietary supplement taken more frequently by 
the treatment of liver diseases such as toxic hepatitis, fatty liver, ischemic injury, cirrhosis, 75 viral hepatitis and radiation toxicity (Luper 1998). Both milk thistle and its active ingredient 76 silymarin are pharmacologically safe and well tolerated (Feher and Lengyel 2012). Silymarin 77 is a lipophilic extract of the milk thistle seeds containing various flavonolignans (which 78 silybin is the major one), has received a tremendous attention over the last decade as an 79 herbal remedy for liver treatment over 30 years. In many cases, the antioxidant properties of 80 silymarin are considered to be responsible for its protective actions (Surai 2015), through 81 alleviating oxidative stress and inflammatory response (Feng et al. 2016). Silymarin is 82 commonly prescribed in cases of viral hepatitis or cirrhosis (Del Prete et al. 2012) because it 83 has hepatoprotective activity (A1-Sayed and Esmat 2016).

Lactulose modifies the bacterial profile in intestinal contents, increasing the lactobacilli and 85 bifidobacteria counts, up-regulating short-chain fatty acids production (Algieri et al. 2014). 86 Lactulose used for the treatment of hepatic encephalopathy(HE) and is effective for primary prevention of overt hepatic encephalopathy in patients with cirrhosis (Sharma et al. 2012). Lactulose decreases the blood endotoxin level and reduce liver damage. It is suggested that lactulose can prevent absorption of endotoxin from the gut and may have an effect on gutderived endotoxemia (Hou 1991).

Liver regeneration evolved presumably to protect wild animals from catastrophic liver loss caused by toxins or tissue injury (Wu et al. 2015). Lactulose administration accelerates post hepatectomized liver regeneration in rats by inducing hydrogen, which may result from 94 attenuation of the oxidative stress response and excessive inflammatory response (Yu et al. 
same time Cetinkunar and Tokgoz et al. demonstrated that silymarin does not contributed to

proliferative regeneration of the liver-which has very important metabolic functions -after

101

partial hepatectomy; instead it will decrease serum levels of transaminases (Cetinkunar et al.

102

2015).

Therefore, for clarifying this confliction we designed present study aimed to investigate the

potential curative effect of silymarin, lactulose or a combination of both against TAA- 105

induced liver cirrhosis in rats.

Formaldehyde solution was purchased from (Dr. Mojallali Industrial chemical complex Co., were purchased from (alfasa, Holland), silymarin from the fruit of Silybum marianum extract was purchased from (SIGMA-ALDRICH, USA), and thioacetamide, hydrochloric acid fuming and 2- thiobarbituric acid were purchased from (Merck, Germany) and trichloroacetic

Animals

All the animal experiments were conducted in accordance the Guide for the Care and Use of Laboratory Animals (1996, published by National Academy Press, 2101 Constitution Ave.

NW, Washington, DC 20055, USA) and approved by the Bu- Ali Sina research committee.

The animals were kept in an agreeable environment in transparent plastic cages under 
Liver cirrhosis was induced according to the method of Li et al. (Li et al. 2002) and Laleman

et al. (Laleman et al. 2006). Thirty-two male Wistar rats were administered by TAA with

Experimental diet was prepared by $50 \mathrm{mg} / \mathrm{kg} /$ day silymarin (Alshawsh et al. 2011), mixed into their chow before forming it into pellets weekly for 8 weeks (Toyoda-Hokaiwado et al.

Rats were divided randomly into five groups: (Table 1) Control group, TAA group: TAA-

The following endpoints were evaluated: (1). Liver injury: Serum alanine amino 
$(A S T)=$ Glutamic Oxal acetic Transaminase (SGOT) and alkaline phosphatase (ALP). (2). 150

Liver regeneration: Calculating liver index (LI) according to the following formula: liver 151

index $=$ liver weight $(\mathrm{g}) /$ bodyweight $(\mathrm{g}) \times 100 \%$; (3). Oxidative stress: Malondialdehyde 152

(MDA) (Amin et al. 2012; Gu et al. 2011a; Gu et al. 2011b) .

At the end of the experiment, all rats were fasted for $12 \mathrm{~h}$ then $5-8 \mathrm{ml}$ whole blood samples

were taken directly from the heart under ketamine $10 \%$ - xylazine $2 \%$ anesthesia. The blood

samples were centrifuged at $10000 \mathrm{rpm}$ for $20 \mathrm{~min}$ for serum separation. All animals were

\section{Biochemical analysis}

Biochemical analysis in serum were done for determination $A L T, A S T$ and $A L P$, serum total

bilirubin, total protein (Pro), albumin (ALB) and gamma glutamyl transferase (GGT) levels

(Roche Hitachi 912 Chemistry Analyzer, Japan) using commercial kits (Man, Iran) for total

At the end of the experiment, all of the rats were decapitated and the liver was rapidly 
MDA measurement has been used frequently to measure lipid peroxidation. The lipid peroxidation assay was performed by determining the MDA, which is the end product of lipid peroxidation, and reacts with thiobarbituric acid (TBA) as a thiobarbituric acid reactive 176 substance (TBARS) to produce a red colored complex that has a peak absorbance (A) at 535 nm. A mixture of trichloroacetic acid (TCA), TBA, and $\mathrm{HCl}$ were added to $1 \mathrm{~mL}$ of serum, and the mixture was heated for $45 \mathrm{~min}$ in a boiling water bath. After cooling and centrifuging it at $1000 \mathrm{~g}$ for $10 \mathrm{~min}$, the cleared supernatant absorbance was measured using SECOMAM

Formalin $10 \%$ fixed liver tissues were embedded in paraffin, and then they were cut into 5

(H\&E) and masson's trichrome protocol, mounted with neutral resin, and examined with

Liver samples stained with $H \& E$ and trichrome masson were assessed for total and individual activity parameters guided by ishak's histological activity index (Ishak et al. 1995). Classified according to the following parameters: confluent necrosis $(0-6)$, periportal or periseptal interface hepatitis (piecemeal necrosis) (0-4), portal inflammation (0-4), focal (spotty) lytic 194 necrosis, apoptosis and focal inflammation (0-4), and architectural changes, fibrosis and 195 cirrhosis (0-6). Additional features which were noted but not scored: bile-duct inflammation, 196 damage and proliferation and steatosis, mild, moderate or marked. 
All data were presented as mean $\pm \mathrm{SD}$. The analysis was carried out using Statistical Package 200

for the Social Sciences (SPSS) version 22. Statistical analyses were conducted by One-way 201

Analysis of Variance (ANOVA) and followed by Least Significant Difference (LSD) 202

analyses to compare various groups with each other. Statistical analyses for scoring of 203

histological parameters were done by Mann- Whitney $U$ from two independent sample tests. 204

Significant differences were analyzed at $P<0.05$. 205

206

$\begin{array}{ll}\text { Results } & 207\end{array}$

$\begin{array}{ll}\text { Mortalities and liver function } & 208\end{array}$

During the whole experimental period, there were minor rat deaths. A total of three rats died: 209

one rat died from the normal group; two rats died in making cirrhotic model. The mortality 210

rate was not more than 7.5\% which makes the survival rate of the model 92.5\%. (Table 1). $\quad 211$

Levels of serum $A L T$ and $A S T$ were assayed as markers of liver injury. thioacetamide 212

produced liver cirrhosis characterized by a significant increase in serum $A L T$ and $A S T$ levels 213

compared with control group. Although thioacetamide have been withdrawn for 8 weeks but 214

in TAA group serum $A L T$ and $A S T$ levels was high and significant in $A S T$ comparing with 215

control group $(P<0.05)$, while treatment with treatments, decreased $A S T$ and $A L T$ levels. 216

Silymarin administration decreased $A S T$ and significantly decreased $A L T$ level compared with $\quad 217$

a TAA group $(P<0.05)$. Lactulose and both silymarin and lactulose significantly decreased 218

liver enzymes $(P<0.01$ and $P<0.05)$ as shown in (Figure 2, A and B). 219

The plasma $A L P$ level of the thioacetamide group increased significantly $(P<0.001)$ when $\quad 220$

compared with the control group (Figure 2C). ALP levels was significantly decreased in SM, 221

LAC and SM+LAC groups by comparing with TAA group. 222 
Liver dysfunction improved upon thioacetamide withdrawal and treatment administration, 223 with decrease of $A L T, A S T$ and $A L P$ it almost resume to the levels as that of the healthy rats 224 (Figure 2A, B and C). In the silymarin group, liver dysfunction improved upon thioacetamide 225 withdrawal with decrease of $A L T, A S T$ and $A L P$ and resume to the levels lower as that of the 226 healthy rats in $A L T$. In lactulose group, recovery of liver dysfunction noted to improve after thioacetamide withdrawal as compared with the controls and with the decrease of $A L T$ and $A S T$ resume to the levels lower as that of control rats but $A L P$ did not resume to the levels as that of control rats. In SM + LAC group, recovery of liver dysfunction resume to the levels of 230 control rats in $A S T$ and lower of control rats in $A L T$ but still $A L P$ is higher than control group. There was a significant increase $(P<0.05$ and $P<0.01)$ in serum total bilirubin of lactulose group ( $2 \mathrm{~g} / \mathrm{kg} /$ day) when compared with control, TAA and SM groups. Therefore there are no significant differences between TAA, SM and SM + LAC groups and the control group, as shown in (Table 2).

It seems that after 8 weeks liver dysfunction improved upon thioacetamide withdrawal and 237 liver ability to synthesize the albumin and other proteins resume to the levels as that of the healthy rats so there are no significant differences between all groups and the control group,

Table 3 indicates the effect of silymarin and lactulose and their combination on the GGT 
MDA was measured to evaluate oxidative stress. MDA, as an oxidation product of lipid

peroxidation injury, increased non-significantly in serum after exposure to thioacetamide

249

(Figure 3). Treating with lactulose or silymarin or their combination non-significantly

250

lowered MDA changes, respectively (Figure 3).

251

252

Effects of silymarin and lactulose and their combination on the liver index

After 18-weeks of thioacetamid administration and 8-weeks of treatment, the weight of rats in

254

the TAA group was non-significantly less than rats in control group (Table 3). The weight of

255

rats in LAC and SM+LAC groups non-significantly increased compared with control and

256

TAA groups. Weight of liver in LAC and SM + LAC groups were significantly higher than

257

liver weight in control group $(P<0.05)$.

258

The liver index used to assess liver damage. LIs of LAC and SM+LAC rats increased and 259 remained at significant higher levels to that of the control $(P<0.05)$, except for SM group 260 which wasn't remarkably higher than control group (Figure 4).

Liver index in the thioacetamide group increased compared with those of normal group,

262

while silymarin group was decreased compared with thioacetamide group but wasn't

263

significant. The results showed that hepatomegaly alleviated after the treatments (Figure 4.).

264

265

Making cirrhotic model

Thioacetamide-induced cirrhosis was established at week 18 after administration 267 thioacetamide, confirmed both macro and microscopically (Figure 5.). Thioacetamide 268 increased amount of collagen deposition in liver tissue stained with masson's trichrome 269 significantly. 
The liver tissue has examined by light microscope for histopathological studies. For 273 determination of liver injury histological examinations have done on sections stained with 274 H\&E and masson stain. For clarifying the extent of hepatic cirrhosis induced by 275 thioacetamide, masson's trichrome had used for liver sections staining. The amount of 276 collagen deposition has increased significantly by thioacetamide in liver tissue, which stained with masson trichrome. Cirrhotic pattern of affected liver was micro nodular, macro nodular, abnormal polyhedron architecture collagen fiber hyperplasia and liver cell necrosis in portal area that extended into the surrounding liver lobules, this leads to criteria of hepatic cirrhosis.

Effect of silymarin and lactulose and their combination on hepatic necroinflammatory

Examination of TAA group showed moderate to severe necroinflammatory activity with

average score of (8.86) with marked confluent necrosis and moderate apoptosis and focal

\section{DISCUSSION}

Key to the first step was to making a cirrhotic animal model. Prolonged exposure to 295 thioacetamide has been reported to result in liver cirrhosis. The toxin affects both perivenular 296 and periportal areas. This model develops macro nodular cirrhosis with portal hypertension, 
the instauration of overt hyper dynamic circulation, associated with the typical histological, 298 biochemical and hemodynamic changes observed in man (Laleman et al. 2006), fibrosis 299 remains stable for weeks after thioacetamide withdrawal (Abraldes et al. 2006). In this study, 300 cirrhotic rat model was successfully developed via drinking thioacetamide water for 18 weeks and was confirmed by histological evidence.

Analysis of histopathology (H\&E staining and masson staining) showed that structure of rat 303 liver in the control group was integrity, the central vein was visible, liver cells were arranged radially and neatly, and lobular contour was clear. However, in liver cirrhosis group the structure of rat liver was abnormal, hepatic lobular architecture was destroyed, pseudo lobule is formed, collagen deposition was around the portal region and connects to each other to form nodules and distort the architecture, and extensive fibrosis with broad septa was observed (Figure 5). These results definitely indicated that the rat model of liver cirrhosis was successfully established.

Thioacetamide-induced hepatotoxic effects characterized by confluent necrosis, piecemeal necrosis, apoptosis and focal inflammation and focal (spotty) lytic necrosis on tissue levels

(Figure 5) which reflected in our study by significant elevation of serum liver enzymes

(Figure 2) and GGT (Table 2) as well as increased collagen deposition and fibrotic areas thioacetamide - induced liver fibrosis and cirrhosis in rats (Gu et al. 2011a; Gu et al. 2011b;

In order of interfere avoidance of thioacetamide with our observation endpoints, 320 thioacetamide has withdrawn before the treatment, which was based on the following 
cirrhotic liver after treatment. The existence of thioacetamide, a hepatic toxin, would

interfere, which would obscure treatment induced regeneration. In general, when used in

324

combination with treatment, it would change treatments effect. Because the treatment period

325

was as long as 56 days, thioacetamide continuously feeding up to 56 days would make most

326

of the rats develop cholangiocarcinoma (Abraldes et al. 2006; De Minicis et al. 2013; Moreira

et al. 1995) or die of thioacetamide toxicity. Upon thioacetamide withdrawal, hepatic

cirrhosis continuously resolve, but persisted up to 120 days, and liver regeneration

significantly decelerates (Gu et al. 2011b). Hence, we have enough time to make treatment on

330

cirrhotic rats. In the literature, withdrawal of thioacetamide prior to the study was also

Thioacetamide had been confirmed to induce hepatic injury and trigger proliferation of liver,

noticeably increased the ratio of liver to overall body weight as presented in LI, which used

to scrutinize potential changes in the liver size, in comparison with control rats (Figure 4).

Thioacetamide, a potent centrilobular hepatotoxicant, undergoes a two-step bio activation

mediated by microsomal CYP2E1 to thioacetamide sulfoxide (TASO), and further to

thioacetamide -S, S-dioxide (TASO2), a reactive metabolite that initiates cellular necrosis

(Chilakapati et al. 2005). Thioacetamide provokes a remarkable increase in the activity and

amount of total mRNA, as well as in the size of the nucleoli of liver cells. During the

biotransformation of thioacetamide both cytochrome $\mathrm{P} 450$ and flavin-containing

monooxygenase (FMO) reduce dioxygen to superoxide anion, which is catalyzed to form

hydrogen peroxide. Therefore, biotransformation of thioacetamide precedes oxidative

damage associated liver injury. This had been already implicated in the disappearance of 
one of the several products formed throughout polyunsaturated fatty acids breakdown

induced by radicals (Lefevre et al. 1997). Silymarin and lactulose alone or in combination

349

protected the liver from oxidative stress caused by thioacetamide as they decreased

350

significantly the elevated serum MDA (Figure 3). In this study, oxidative stress after cirrhosis

351

manifested as increased lipid peroxidation. After silymarin treatment silymarin acts as a

352

scavenger of free radicals, so it decreases oxidative stress. The antioxidative property of

353

hydrogen might play a main role in the molecular mechanism of lactulose-enhanced liver

354

regeneration.

355

GGT is one of the important markers of liver injury, and their elevation discloses cholestasis

356

and bile duct necrosis. When liver cells are damaged, the serum $A S T$ and $A L T$ are released

357

into the blood from the cells. Therefore, $A L T$ and $A S T$ are two of the most reliable markers of

358

necrosis or hepatocellular injury. Data from the present study have demonstrated that SM and

359

LAC and SM+LAC groups protected liver from tissue inflammation, which exhibited an

360

obvious decrease in serum markers of liver function brought back these enzymes to near

361

routine level by possibly preserving the functional integrity of the hepatocytes, showing their

362

defense action against thioacetamide -induced hepatotoxicity as compared to the model

363

control (TAA group) (Figure 2). Silymarin decreased serum GGT level but it didn't happen in

364

LAC and SM+LAC groups (Table 3). Treatments decreased fibrosis scores in LAC and

SM+LAC groups compared to TAA group although it wasn't significant due to the short time

of observations. (Data not shown) (Figure 5) indicate effectiveness of silymarin, lactulose or

367

both in the recovery of liver function after cellular damage. Amin et al. and Fadillioglu et al.,

368

respectively (Amin et al. 2012; Fadillioglu et al. 2010) demonstrated that silymarin and

369

lactulose significantly reduced the increased $A L T$ and $A S T$ caused by thioacetamide. These

370

are evidence for the important role of silymarin and lactulose alone or in combination in

371

protecting cells from lipid peroxidation and restoring cell membrane function so they have

372 
hepatocurative effects. In accordance to these results, observations indicated that an increase

in serum liver enzymes levels in TAA group may be result of massive necrosis and late stage

of fibrosis (Gu et al. 2011a; Gu et al. 2011b). The elevation of the serum activities of $A L P$

375

and GGT indicated the induction of cholestasis (Kawai et al. 2009) while decreased serum

376

liver enzymes activities in SM and LAC groups may be due to recovery, as decreasing serum

377

liver enzymes activities in hepatic injury occur with recovery, given that lactulose has a

378

function of reducing blood ammonia levels, and hydrogen produced by anaerobic organisms

379

was the key for its therapeutic effects.

380

We found that silymarin, lactulose and their combination have dramatic effects on MDA, as

381

serum MDA significantly decreased. The probable antioxidant mechanisms of silymarin are

382

direct scavenging free radicals and chelating free $\mathrm{Fe}$ and $\mathrm{Cu}$, preventing free radical

383

formation, improving the integrity of mitochondria's electron transport chain in stress

conditions, maintaining an optimal redox balance in the cell, activating vitagenes, and

providing additional protection in stress conditions, affecting the microenvironment of the

gut, have hepatoprotective effects, decreasing inflammatory responses, is an emerging

387

mechanism of silymarin protective effects in liver toxicity and various liver diseases (Surai

388

2015). The exposure of hepatocytes to free fatty acids in the context of dyslipidemia,

389

resulting in increased ROS production and mitochondrial damage (Tarantino et al. 2010),

rapid fermentation of lactulose into organic acids at rates exceeding colonic buffering

capacity to reduce formation of toxic fatty acids and ammonia from amino acids,

392

polypeptides, and blood in the colon maybe is the probable mechanism of lactulose (Bonnkn

393

1990). 
In conclusion, our findings of this study are: (1) As proved by serum markers for liver 397

function and histological findings, silymarin, lactulose and silymarin \& lactulose combination $\quad 398$

could protect against thioacetamide-induced liver cirrhosis in rats. (2) Silymarin \& lactulose 399

combination should be useful for the treatment of liver cirrhosis at physiological 400

concentrations. (3) Silymarin, lactulose, and silymarin \& lactulose combination exert 401

significant curative effects against thioacetamide -induced oxidative stress and liver damage $\quad 402$

by augmenting host antioxidant defense mechanisms. (4) Silymarin \& lactulose combination 403

curative effects are due to increase of antioxidative effects as well as lowering the extent of 404

$\begin{array}{ll}\text { lipid peroxidation. } & 405\end{array}$

406

$\begin{array}{ll}\text { Declarations } & 407\end{array}$

$\begin{array}{ll}\text { Ethics approval and consent to participate } & 408\end{array}$

All the animal experiments were conducted in accordance with the guideline and approved by the $\mathrm{Bu}-\quad 409$

$\begin{array}{ll}\text { Ali Sina research committee. } & 410\end{array}$

411

$\begin{array}{ll}\text { Consent for publication } & 412\end{array}$

$\begin{array}{ll}\text { Not applicable. } & 413\end{array}$

414

$\begin{array}{ll}\text { Availability of data and materials } & 415\end{array}$

The datasets during and/or analysed during the current study available from the 416 $\begin{array}{ll}\text { corresponding author on reasonable request. } & 417\end{array}$

$\begin{array}{ll}\text { Competing interests } & 419\end{array}$

$\begin{array}{ll}\text { The authors declare that they have no competing interests. } & 420\end{array}$ 
Funding

This work was supported by research council of Bu-Ali Sina University (Grant No. 94-379).

423

424

Authors' contributions

425

Mozhgan Ghobadi Pour: Done the entire project.

426

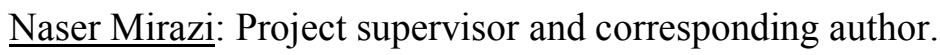

427

Hojjatollah Alaei: Supervisor assistant, project done in Dr. Alaei’s lab and he provide all 428

what was needed.

429

Shirin Moradkhani: Proofreading the article.

430

Ziba Rajaei: Supervised the MDA test.

431

Alireza Monsef Esfahani: Professor in Pathology histopathological expert.

432

433

Acknowledgements

We thank Dr. Zahra Rad Ahmadi for providing help during the research.

Collaborating author names:

436

Mozhgan Ghobadi Pour, Naser Mirazi, Hojjatollah Alaei, Shirin Moradkhani, Ziba Rajaei,

437

Alireza Monsef Esfahani.

438

439

References:

Abraldes, J. G., Pasarín, M., and García-Pagán, J. C. 2006. Animal models of portal hypertension.

Al-Sayed, E.,and Esmat, A. 2016. Hepatoprotective and antioxidant effect of ellagitannins and galloyl 
Algieri, F., Rodríguez-Nogales, A., Garrido-Mesa, N., Vezza, T., Garrido-Mesa, J., Utrilla, M. P., et

al. 2014. Intestinal anti-inflammatory effects of oligosaccharides derived from lactulose in the

trinitrobenzenesulfonic acid model of rat colitis. J. Agric. Food Chem. 62(19): 4285-4297.

449

Alshawsh, M. A., Abdulla, M. A., Ismail, S., and Amin, Z. A. 2011. Hepatoprotective effects of

450

Orthosiphon stamineus extract on thioacetamide-induced liver cirrhosis in rats. Evid. Based

451

Complement. Alternat. Med. 2011:103039. doi: 10.1155/2011/103039

452

Amin, Z. A., Bilgen, M., Alshawsh, M. A., Ali, H. M., Hadi, A. H. A., and Abdulla, M. A. 2012.

453

Protective role of Phyllanthus niruri extract against thioacetamide-induced liver cirrhosis in rat

454

model. Evid. Based Complement. Alternat. Med. 2012: 157456.

455

Bonnkn, H. 1990. The degradation of amino acids, proteins, and blood to short-chain fatty acids in 456

colon is prevented by lactulose. Gastroenterology, 98(2): 353-360.

457

Cetinkunar, S., Tokgoz, S., Bilgin, B. C., Erdem, H., Aktimur, R., Can, S., et al. 2015. The effect of

458

silymarin on hepatic regeneration after partial hepatectomy: is silymarin effective in hepatic

459

regeneration? Int. J. Clin. Exp. Med. 8(2): 2578.

460

Chilakapati, J., Shankar, K., Korrapati, M. C., Hill, R. A.,Mehendale, H. M. 2005. Saturation

461

toxicokinetics of thioacetamide: role in initiation of liver injury. Drug Metab. Dispos. 33(12): 1877-

1885.

462

463

Constantinou, M. A., Theocharis, S. E., and Mikros, E. 2007. Application of metabonomics on an

464

experimental model of fibrosis and cirrhosis induced by thioacetamide in rats. Toxicol. Appl.

465

Pharmacol. 218(1): 11-19.

466

De Minicis, S., Kisseleva, T., Francis, H., Baroni, G. S., Benedetti, A., Brenner, D., et al. 2013. Liver

467

carcinogenesis: rodent models of hepatocarcinoma and cholangiocarcinoma. Dig. Liver Dis. 45(6):

468

450-459.

469

Del Prete, A., Scalera, A., Iadevaia, M. D., Miranda, A., Zulli, C., Gaeta, L., et al. 2012. Herbal

470

products: benefits, limits, and applications in chronic liver disease. Evid. Based Complement.

471

Alternat. Med. 2012: 837939.

472 
Fadillioglu, E., Gursul, C., and Iraz, M. 2010. Effects of caffeic acid phenethyl ester on

thioacetamide-induced hepatic encephalopathy in rats. Prog. Neuropsychopharmacol. Biol.

474

Psychiatry, 34(8): 1440-1445.

475

Feher, J., and Lengyel, G. 2012. Silymarin in the prevention and treatment of liver diseases and 476 primary liver cancer. Curr. Pharm. Biotechnol. 13(1): 210-217.

477

Feng, B., Meng, R., Huang, B., Shen, S., Bi, Y., and Zhu, D. 2016. Silymarin alleviates hepatic 478 oxidative stress and protects against metabolic disorders in high-fat diet-fed mice. Free Radic. Res. 50(3): 314-327.

480

Gu, K., Lai, S.-T., Ma, N.-Y., Zhao, J.-D., Ren, Z.-G., Wang, J., et al. 2011a. Hepatic regeneration 481 after sublethal partial liver irradiation in cirrhotic rats. J. Radiat. Res. (Tokyo), 52(5): 582-591.

Gu, K., Zhao, J.-D., Ren, Z.-G., Ma, N.-Y., Lai, S.-T., Wang, J., et al. 2011b. A natural process of 483 cirrhosis resolution and deceleration of liver regeneration after thioacetamide withdrawal in a rat model. Mol. Biol. Rep. 38(3): 1687-1696.

Hou, R. 1991. [Treatment of gut-derived endotoxemia with lactulose. An experimental study]. [article 485 in Chinese]. Zhonghua Wai Ke Za Zhi, 29(4): 248-250, 272.

486

Ishak, K., Baptista, A., Bianchi, L., Callea, F., De Groote, J., Gudat, F., et al. 1995. Histological 487 grading and staging of chronic hepatitis. J. Hepatol. 22(6): 696-699.

Kawai, H., Ishibashi, T., Kudo, N., Kawashima, Y.,and Mitsumoto, A. 2012. Behavioral and 489 biochemical characterization of rats treated chronically with thioacetamide: proposal of an animal model for hepatic encephalopathy associated with cirrhosis. J. Toxicol. Sci. 37(6): 1165-1175.

Kawai, H., Kudo, N., Kawashima, Y., and Mitsumoto, A. 2009. Efficacy of urine bile acid as a non493 invasive indicator of liver damage in rats. J. Toxicol. Sci. 34(1): 27-38.

Kumar, A. E., Susmitha, K., Swathy, B., Ramu, E., and Venkatesh, B. 2014. A review on liver 495 disorders and screening models of hepatoprotective agents. Int. J. Allied Med. Sci. Clin. Res. 2(2): 496 136-150.

Laleman, W., Elst, I. V., Zeegers, M., Servaes, R., Libbrecht, L., Roskams, T., et al. 2006. A stable 498 model of cirrhotic portal hypertension in the rat: thioacetamide revisited. Eur. J. Clin. Invest. 36(4): 
Lefevre, G., Beljean-Leymarie, M., Beyerle, F., Bonnefont-Rousselot, D., Cristol, J., Therond, P., et 501

al. 1997. [Evaluation of lipid peroxidation by measuring thiobarbituric acid reactive substances], Ann.

502

Biol. Clin. (Paris), Vol. 56, pp. 305-319.

503

Li, X., Benjamin, I. S., and Alexander, B. 2002. Reproducible production of thioacetamide-induced

504

macronodular cirrhosis in the rat with no mortality. J. Hepatol. 36(4): 488-493.

505

Luper, S. 1998. A review of plants used in the treatment of liver disease: part 1. Altern. Med. Rev.

506

3(6): 410-421.

507

Moreira, E., Fontana, L., Periago, J. L., De Medina, F. S., and Gil, Á. 1995. Changes in fatty acid

508

composition of plasma, liver microsomes, and erythrocytes in liver cirrhosis induced by oral intake of

509

thioacetamide in rats. Hepatology, 21(1): 199-206.

510

Prasad, R., Naime, M., Routray, I., Mahmood, A., Khan, F., and Ali, S. 2010. Valeriana jatamansi

511

partially reverses liver cirrhosis and tissue hyperproliferative response in rat. Methods Find. Exp.

512

Clin. Pharmacol. 32(10): 713.

513

Rajaei, Z., Hadjzadeh, M.-A.-R., Nemati, H., Hosseini, M., Ahmadi, M., and Shafiee, S. 2013. 514

Antihyperglycemic and antioxidant activity of crocin in streptozotocin-induced diabetic rats. J. Med. 515

Food, 16(3): 206-210.

516

Schuppan, D., and Afdhal, N. H. 2008. Liver cirrhosis. The Lancet, 371(9615): 838-851.

517

Sharma, P., Sharma, B. C., Agrawal, A., and Sarin, S. K. 2012. Primary prophylaxis of overt hepatic

518

encephalopathy in patients with cirrhosis: an open labeled randomized controlled trial of lactulose

519

versus no lactulose. J. Gastroenterol. Hepatol. 27(8): 1329-1335.

520

Surai, P. F. 2015. Silymarin as a Natural Antioxidant: An Overview of the Current Evidence and

521

Perspectives. Antioxidants, 4(1): 204-247.

522

Tarantino, G., Citro, V., Esposito, P., Giaquinto, S., de Leone, A., Milan, G., et al. 2009. Blood

523

ammonia levels in liver cirrhosis: a clue for the presence of portosystemic collateral veins. BMC

524

Gastroenterol. 9(1): 1 .

525

Tarantino, G., Colao, A., Capone, D., Conca, P., Tarantino, M., Grimaldi, E., et al. 2010. Circulating

526

levels of cytochrome C, gamma-glutamyl transferase, triglycerides and unconjugated bilirubin in

527 
overweight/obese patients with non-alcoholic fatty liver disease. J. Biol. Regul. Homeost. Agents, 528

25(1): $47-56 . \quad 529$

Toyoda-Hokaiwado, N., Yasui, Y., Muramatsu, M., Masumura, K., Takamune, M., Yamada, M., et al. 530

2011. Chemopreventive effects of silymarin against 1, 2-dimethylhydrazene plus dextran sodium 531

sulfate-induced inflammation-associated carcinogenicity and genotoxicitiy in the colon of gpt delta 532

rats. Carcinogenesis, 32(10):1512-7. doi: 10.1093/carcin/bgr130. 533

Wiegand, J., and Berg, T. 2013. The etiology, diagnosis and prevention of liver cirrhosis: part 1 of a 534

series on liver cirrhosis. Deutsches Ärzteblatt International, 110(6): 85.

Wu, J.-P., Tsai, C.-C., Yeh, Y.-L., Lin, Y.-M., Lin, C.-C., Day, C. H., et al. 2015. Silymarin 536

Accelerates Liver Regeneration after Partial Hepatectomy. Evid. Based Complement. Alternat. Med. 537

2015: $603529 . \quad 538$

Yu, J., Zhang, W., Zhang, R., Ruan, X., Ren, P.,and Lu, B. 2015. Lactulose accelerates liver 539

regeneration in rats by inducing hydrogen. J. Surg. Res. 195(1): 128-135. 540

Zhou, W.-C., Zhang, Q.-B., and Qiao, L. 2014. Pathogenesis of liver cirrhosis. World J. 541

Gastroenterol. 20(23): 7312. 
Table 1- Experiment design and mortalities.

\begin{tabular}{lccc}
\hline Experiment groups & Mortalities & Subgroups(7 rats) & Dose \\
\hline Healthy rats(8 rats) & $1 / 8$ & Control & - \\
Cirrhotic rats & $2 / 32$ & Negative Control & - \\
& - & Silymarin & $50 \mathrm{mg} / \mathrm{kg} / \mathrm{day}$ \\
& - & Lactulose & $2000 \mathrm{mg} / \mathrm{kg} / \mathrm{day}$ \\
& - & Silymarin $\&$ & $50 \mathrm{mg} / \mathrm{kg} / \mathrm{day}$ \\
& & Lactulose & $+2000 \mathrm{mg} / \mathrm{kg} / \mathrm{day}$ \\
\hline
\end{tabular}

Table 2- Effect of thioacetamide, silymarin, lactulose alone or in combination on some serum parameters.

\begin{tabular}{|c|c|c|c|c|c|}
\hline Group & $\begin{array}{l}\text { Number } \\
\text { of animals }\end{array}$ & $\begin{array}{l}\text { Total bilirubin } \\
\text { (mg/dl) }\end{array}$ & $A L B(\mathrm{~g} / \mathrm{dl})$ & $\begin{array}{l}\text { Total protein } \\
\text { (g/dl) }\end{array}$ & $G G T(I U / L)$ \\
\hline Control & 7 & $0.227 \pm 0.048$ & $3.141 \pm 0.434$ & $6.200 \pm 1.184$ & $3.86 \pm 3.185$ \\
\hline TAA & 7 & $0.197 \pm 0.011$ & $3.498 \pm 0.202$ & $7.004 \pm 0.629$ & $8.14 \pm 5.640 *$ \\
\hline SM & 7 & $0.198 \pm 0.052$ & $3.088 \pm 0.433$ & $6.085 \pm 0.915$ & $6.14 \pm 5.336$ \\
\hline LAC & 7 & $0.285 \pm 0.050 * \# \# \$$ & $3.078 \pm 0.531$ & $5.912 \pm 1.469$ & $8.14 \pm 3.671$ \\
\hline $\mathbf{S M}+\mathbf{L A C}$ & 7 & $0.225 \pm 0.023 ¥$ & $2.915 \pm 0.760 \#$ & $5.590 \pm 1.708 \#$ & $10.86 \pm 7.603 *$ \\
\hline \multicolumn{6}{|c|}{ Note: Values represent mean \pm SD. Values are significantly different $(* P<0.05$ vs. Control), $(\# P<0.05$ vs. } \\
\hline
\end{tabular}


Table 3- Effects of treatments on body and liver weights of rats for 8 weeks.

\begin{tabular}{|c|c|c|c|}
\hline Groups & Dose (mg/kg/day) & Body weight (g) & Liver weight (g) \\
\hline Control & - & $291.29 \pm 13.671$ & $8.045 \pm 0.535$ \\
\hline TAA & - & $287.57 \pm 16.582$ & $9.320 \pm 1.538$ \\
\hline SM & 50 & $284.57 \pm 13.661$ & $9.140 \pm 1.503$ \\
\hline LAC & 2000 & $297.71 \pm 15.108$ & $10.195 \pm 2.039 *$ \\
\hline $\mathbf{S M}+\mathbf{L A C}$ & $50+2000$ & $299.00 \pm 15.384$ & $10.422 \pm 1.808 *$ \\
\hline
\end{tabular}

Note: Results were presented as the mean \pm SD. $\left({ }^{*} P<0.05\right.$ vs. Control). 
Figure captions

Figure. 1. Schematic representation of the experimental design used for the study.

Figure. 2. Changes of liver function after thioacetamide withdrawal and treatment administration.

571

Effect of (thioacetamide for 18 weeks), silymarin (50 mg/kg daily) and lactulose ( $2 \mathrm{~g} / \mathrm{kg}$ daily) alone

572

or in combination on liver functions by serum $A S T(\mathrm{~A}), A L T(\mathrm{~B})$ and $A L P(\mathrm{C})$ activities. Values: (Mean

573

\pm SD). ${ }^{*} P<0.05$ vs. Control group. ${ }^{* *} P<0.01$ vs. Control group. ${ }^{* * *} P<0.001$ vs. Control group.

574

$\# P<0.05$ vs. TAA group. $\# \# P<0.01$ vs. TAA group. $\# \# P<0.001$ vs. TAA group.

575

Figure. 3. Effect of thioacetamide (for 18 weeks), silymarin (50 mg/kg daily) and lactulose ( $2 \mathrm{~g} / \mathrm{kg}$

576

daily) alone or in combination on serum MDA levels. Values: (Mean \pm SD). $\# P<0.05$ vs. TAA

577

group. \#\# $P<0.01$ vs. TAA group.

578

Figure. 4. Changes of the liver index (LI). In all groups, LIs were higher in all than that of healthy

579

rats. Values: (Mean $\pm \mathrm{SD}) .{ }^{*} P<0.05$ vs. Control group.

580

Figure. 5. The changes in liver photomicrographs of rats. The livers were stained with H\&E and

Masson's trichrome. Central vein (CV), Hepatic cords (H), Portal Vein (PV), periductal Fibrosis

582

$(\mathrm{F})$ and Fatty Change in a diffuse manner $(\mathrm{FC}) .(\rightarrow)$ hepatocyte necrosis, destruction of lobular

583

architecture, and extensive fibrosis. H\&E and Masson's trichrome, magnification 100×.

584

Figure. 6 - Effect of thioacetamide (daily for 18 weeks), silymarin (50 mg/kg daily) and lactulose (2

585

$\mathrm{g} / \mathrm{kg}$ daily) alone or in combination on (A) liver necroinflammatory score and values: (Mean $\pm \mathrm{SD}$ ).

586

** $\mathrm{P}<0.01$ vs. Control group.

587

588

589

590

591

592

593

594

https://mc06.manuscriptcentral.com/cjpp-pubs 


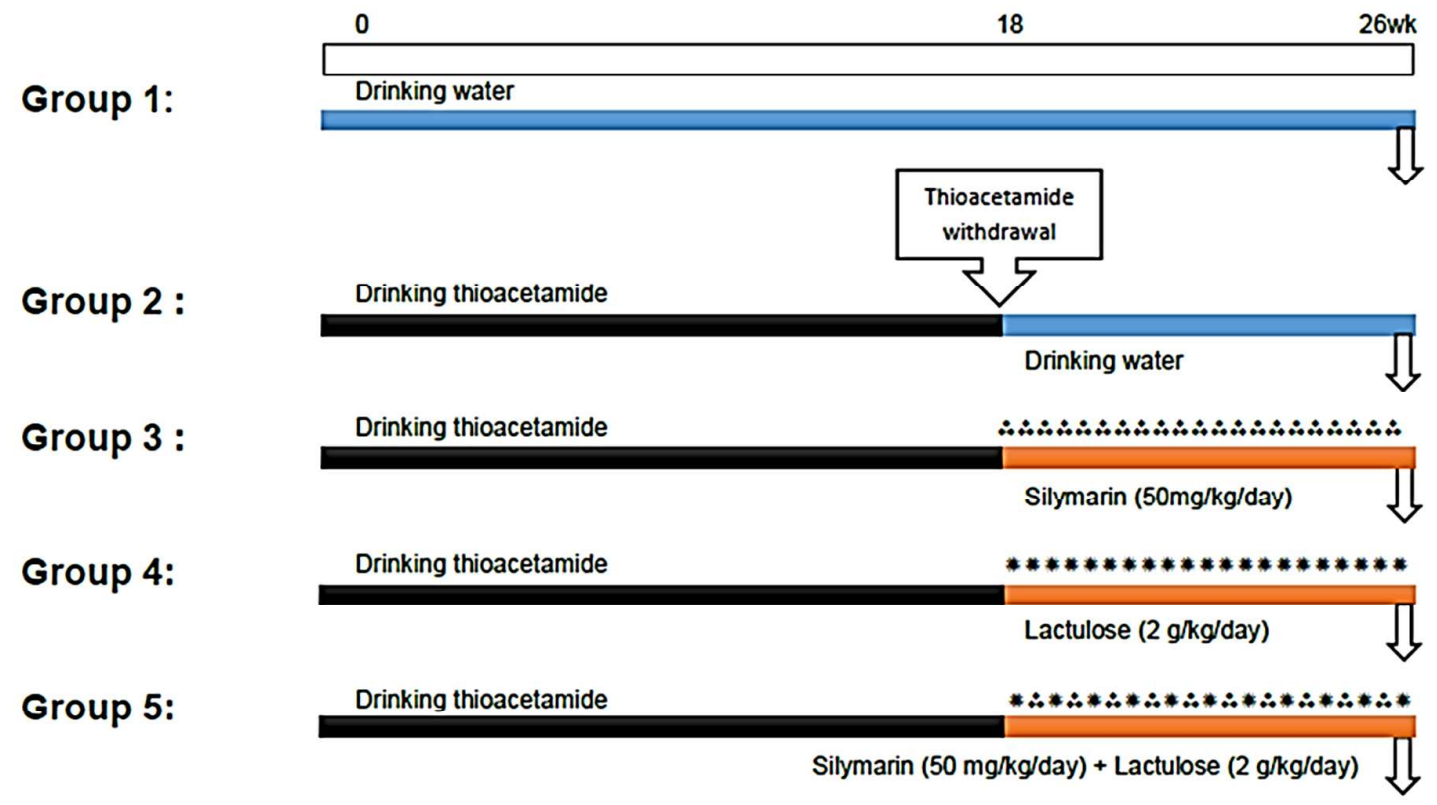

\begin{tabular}{|l|}
\hline Time of sacrifice \\
Drinking water \\
Drinking thioacetamide \\
Drinking or feeding treatment \\
\end{tabular}


Figure 2A

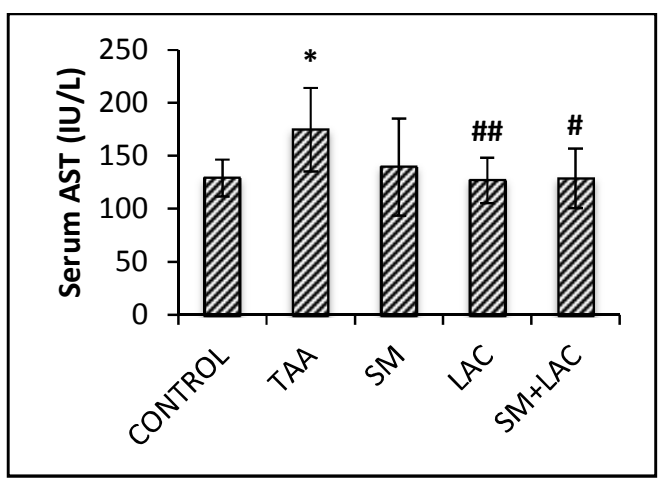

Figure 2B

602

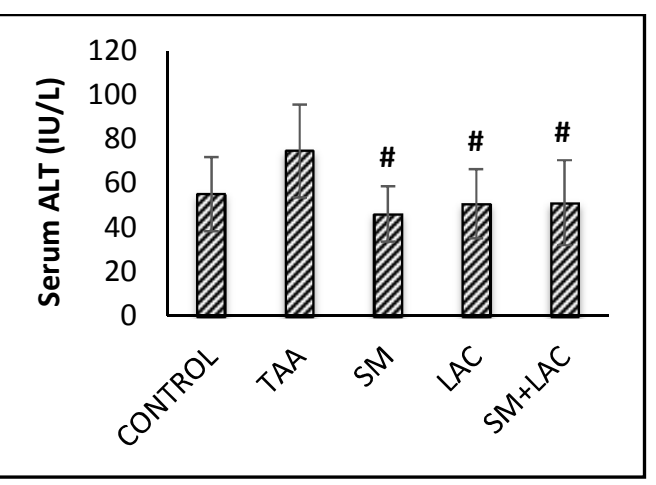

603

Figure 2C

604

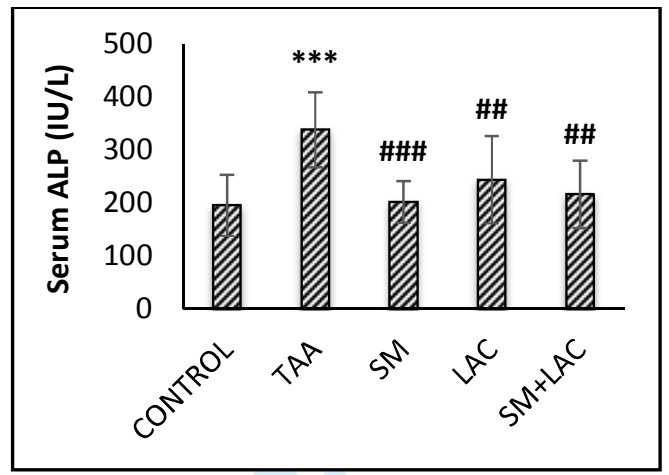

605

606

607 
Figure 3

608

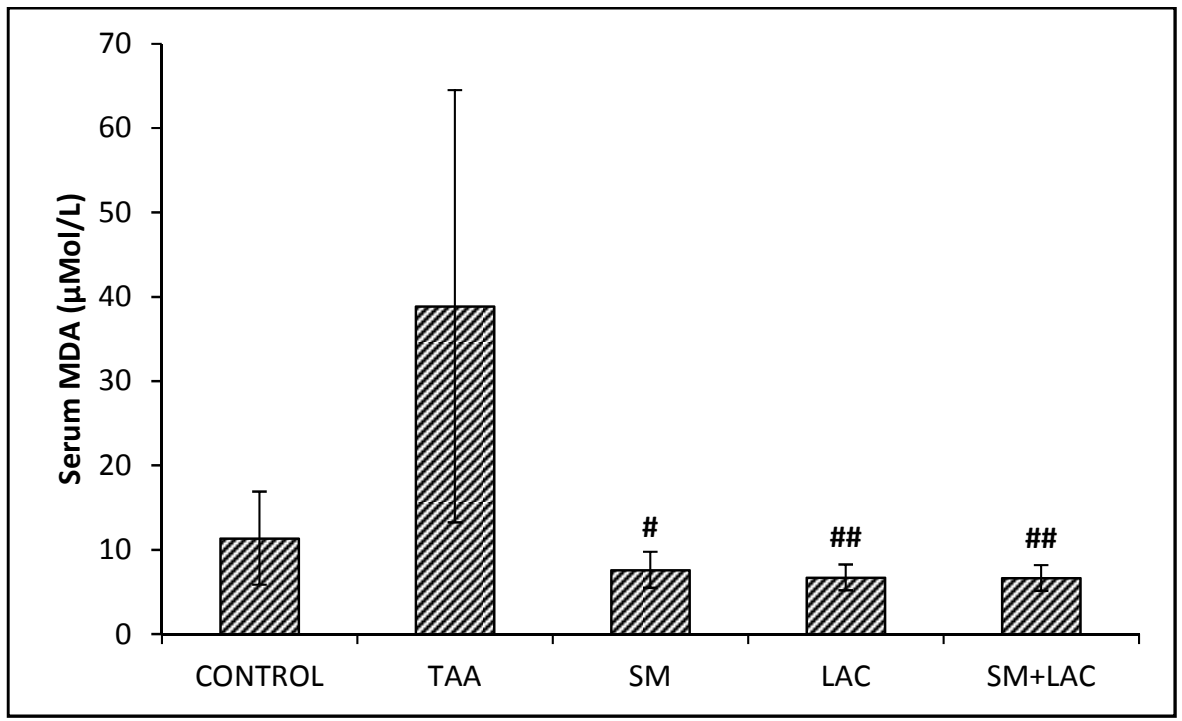

609

610

611 
Figure 4

612

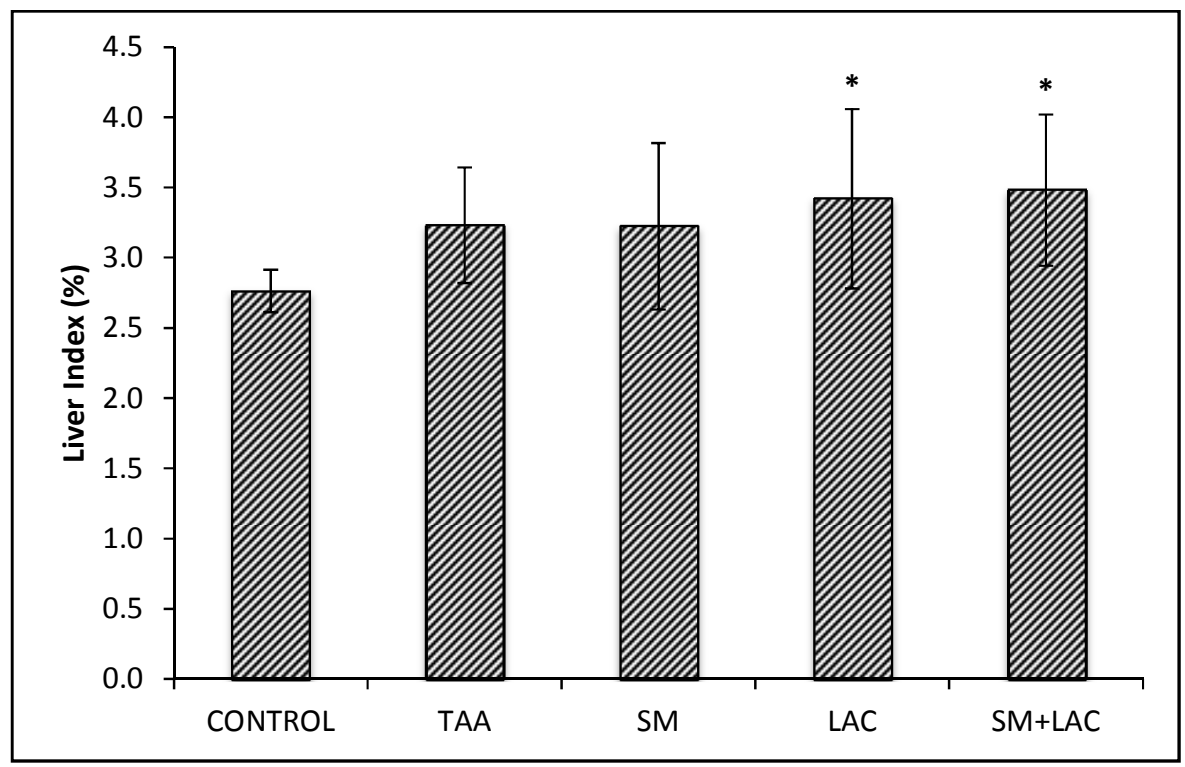

613

614

615

https://mc06.manuscriptcentral.com/cjpp-pubs 
Figure 5
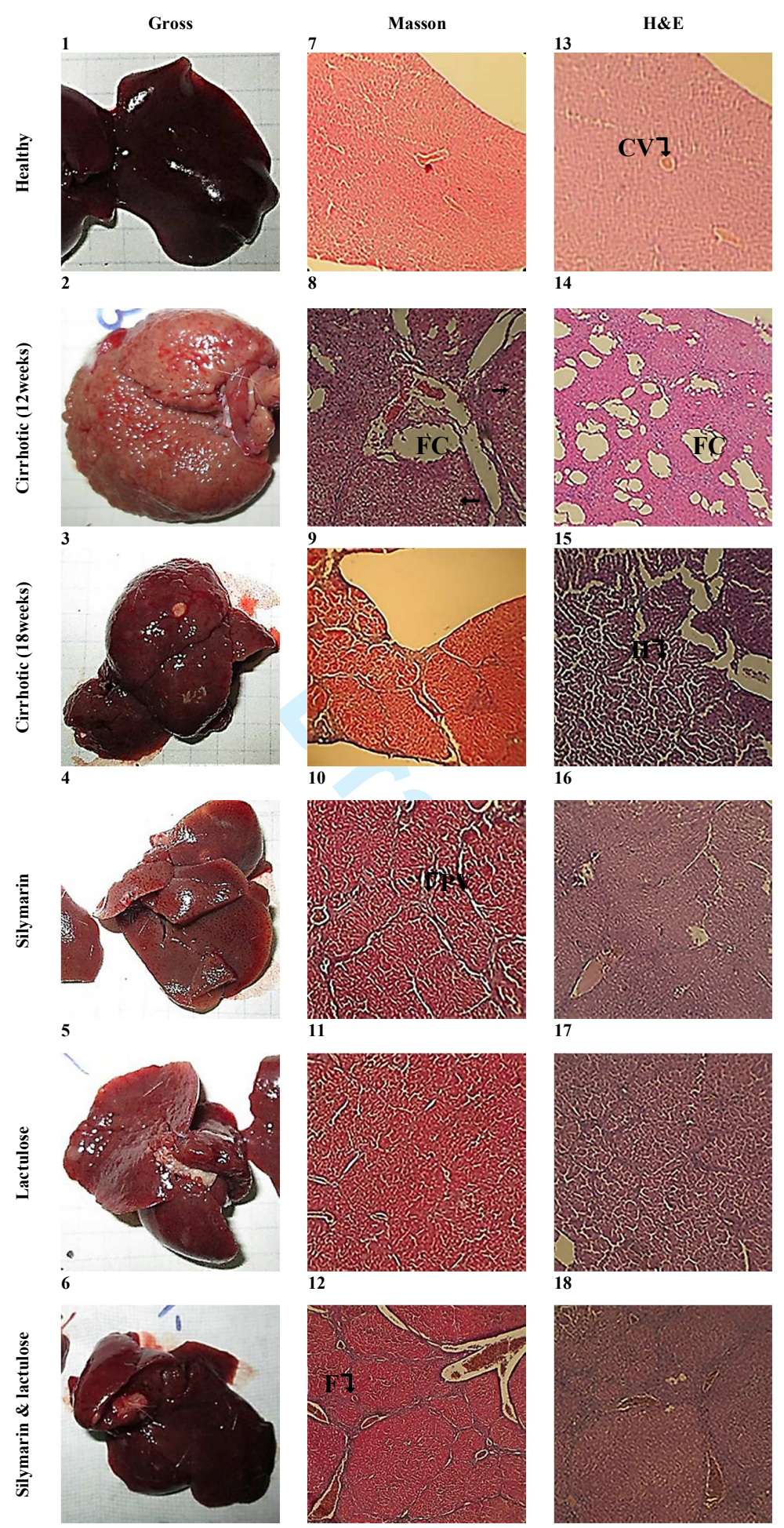
Figure 6

619

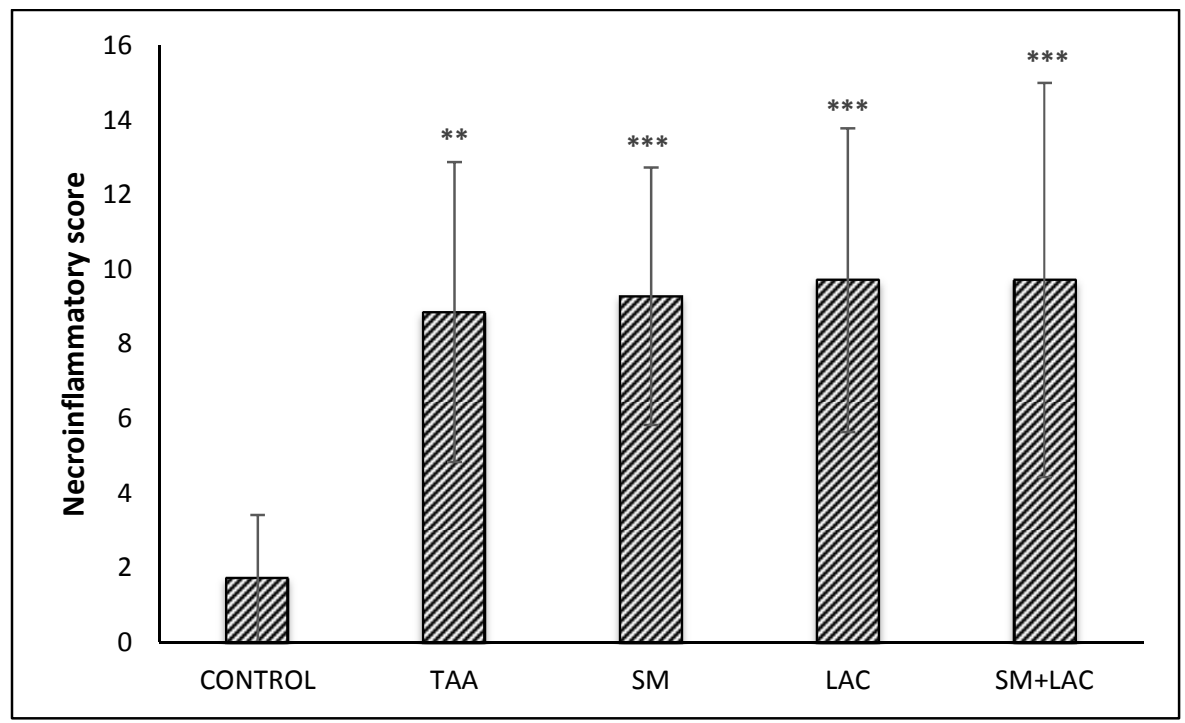

620 